\title{
Comparison of mucin-1 in human breast cancer and canine mammary gland tumor: a review study
}

\author{
Rana Vafaei ${ }^{1}$, Mitra Samadi ${ }^{1}$, Aysooda Hosseinzadeh ${ }^{1}$, Khadijeh Barzaman ${ }^{1,3}$, MohammadReza Esmailinejad ${ }^{4}$, \\ Zohreh Khaki ${ }^{1,2^{*}}$ and Leila Farahmand ${ }^{1 *}$ (B)
}

\begin{abstract}
Mucin-1 (MUC-1) is a transmembrane glycoprotein, which bears many similarities between dogs and humans. Since the existence of animal models is essential to understand the significant factors involved in breast cancer mechanisms, canine mammary tumors (CMTs) could be used as a spontaneously occurring tumor model for human studies. Accordingly, this review assessed the comparison of canine and human MUC-1 based on their diagnostic and therapeutic aspects and showed how comparative oncology approaches could provide insights into translating pre-clinical trials from human to veterinary oncology and vice versa which could benefit both humans and dogs.
\end{abstract}

Keywords: Mucin-1, Canine mammary tumor, Preclinical trials, Immunotherapy

\section{Background}

The increasing demand in cancer research for more suitable immune-competent animal models, which develop cancer spontaneously, raised companion animals with naturally occurring tumors as a valuable resource [1-4]. Although comparative oncology, as a method for accelerating the development of drugs by introducing animal patients, is currently underused, it should continue to make a great opportunity to investigate many aspects of human cancers, from physiology to treatment [2, 5]. Canine mammary tumors (CMTs) are the most common malignancies in female dogs with a high mortality rate (which has been broadly characterized for genetic defects), bearing many common characteristics with human breast cancer (HBC) [6, 7]. Clinical and molecular similarities have been identified between $\mathrm{HBC}$ and CMT $[3,4,7,8]$. Intact female dogs have a greater incidence of

\footnotetext{
*Correspondence: zkhaki@ut.ac.ir; laylafarahmand@gmail.com ${ }^{1}$ Recombinant Proteins Department, Breast Cancer Research Center, Motamed Cancer Institute, ACECR, No.146, South Gandi Ave, Vanak Sq, Tehran, Iran

Full list of author information is available at the end of the article
}

mammary tumors (84\%), of which $78.2 \%$ lead to malignant forms; this may be due to the late submission of samples for histological examination [4]. CMT and HBC are similar in many ways, including environmental, biological, epidemiological, clinical, genetic, and pathological elements [9-11]. For instance, there is a great similarity of molecular and histological heterogeneity between CMT and HBC [9]. Moreover, the similarity between human and canine genome organizations are more than human and mouse [12], which proves that dog could be a better model for human cancer studies. Another notable benefit of using CMT for HBC research is that this cancer is the most common tumor in female dogs, with an incidence rate more than that seen in the human population [7]. Due to the lack of human tissue samples and the presence of ethical limitations in the research area, there is an urgent demand to find in vivo and in vitro alternative models for HBC [13]. Although cell lines are widely used in preclinical and in vitro studies, many believe that they are not a real model of cancer cells [13]. For in vivo studies, xenograft models and engineered dogs, cats, and mice are used [13]. Among them, dogs can be considered original author(s) and the source, provide a link to the Creative Commons licence, and indicate if changes were made. The images or other third party material in this article are included in the article's Creative Commons licence, unless indicated otherwise in a credit line to the material. If material is not included in the article's Creative Commons licence and your intended use is not permitted by statutory regulation or exceeds the permitted use, you will need to obtain permission directly from the copyright holder. To view a copy of this licence, visit http://creativecommons.org/licenses/by/4.0/. The Creative Commons Public Domain Dedication waiver (http://creativeco mmons.org/publicdomain/zero/1.0/) applies to the data made available in this article, unless otherwise stated in a credit line to the data. 
as an optimum animal model [13]. The reason is that many markers, which play a critical role in $\mathrm{HBC}$, have the same role in canine tumorigenesis, such as the mucin family $[13,14]$. Mucins are large, highly glycosylated proteins expressed by various secretory epithelial cells [14]. MUC-1 is also known as the cluster of differentiation 227 (CD-227), cancer antigen 15-3 (CA15-3), and Krebs von den Lungen-6 (KL-6). As the most thoroughly studied tumor-associated antigen (TAA), it is a type 1 transmembrane glycoprotein, which has a high molecular weight [15]. O-glycoprotein mucin is expressed at low levels on the apical borders of normal secretory epithelial cells [16]; it has a linearly extended extracellular domain, most of which contains tandem repeat sequences [17]. Same as other mucins, this glycoprotein is involved in protecting epithelial barrier, cellular adhesion, and lubrication, among other functions [18]. The identification of specific and sensitive molecular biomarkers involved in breast cancer has a consequential clinical significance; hence, MUC-1 has been broadly investigated for the treatment of HBC [19]. Overexpression and aberrantly glycosylation of this glycoprotein is related to the tumor invasion in human carcinomas and results in poor prognosis [20]. Also increasing concentrations of mucin-type glycoproteins in serum are due to increased tumor invasiveness in human [21]. MUC-1 expression is also known to be related to invasiveness and metastasis in CMT and has prognostic value $[8,20]$. Thus, MUC-1 has become an interesting subject in the diagnosis and treatment of CMT and HBC [4, 14, 20, 22-24]. Many scientists believe that CMT is a perfect model for HBC investigations [9, 10]. CMT can be used for the evaluation of immunotherapy and other therapeutic applications [10]. Accordingly, this review drew a comparison between the MUC-1 roles in HBC and CMTs as a model for human studies.

\section{Gene and protein structure of mucin-1}

The human MUC-1 gene of 4-7 kb length consists of seven exons, which can be alternatively spliced to make transcripts from 3.7 to $6.4 \mathrm{~kb}$. This gene encodes a single polypeptide chain containing three different domains, i.e., a short C-terminal cytoplasmic domain (CD), short transmembrane domain (TD), and large $\mathrm{N}$-terminal extracellular domain (ECTO) [25]. Tyrosine-phosphorylated CD is involved in signal transduction [26]. ECTO is made of a variable number (30-100) of 20 amino acids (GVTSAPDTRPAPGSTAPPAH), called variable number of tandem repeats (VNTRs) [18]. VNTR amino acid sequences can differ in various cancer cell lines as a result of the highly polymorphic character of this region. Dissimilar to the human MUC-1 sequence, mouse MUC-1 has 16 amino acid repeating tandems and bears only $34 \%$ sequence identity with its counterpart in humans [27].
An autocatalytic process in the endoplasmic reticulum cleaves this single polypeptide into two subunits as follows: (i) the MUC-1 N-terminal subunit (MUC-1 N-ter, MUC-1-N) that contains VNTRs and weighs more than $250 \mathrm{kDa}$, and (ii) MUC-1 C-terminal subunit (MUC-1 C-ter, MUC-1-C) that includes 58 amino acids of ECTO, whole 28 amino acids of TD, and whole 72 amino acids of $\mathrm{CD}$, all weighing $23 \mathrm{kDa}$ [28]. MUC-1-N is tethered to the cell surface by dimerization with MUC-1-C [28]. A transmembrane cleavage product of MUC-1, known as MUC$1^{*}$, which is the predominant form of this glycoprotein on cancer cells [29], functions as a growth factor receptor on tumor cells [29]. This cleavage of the full-length ECTO and formation of MUC-1* membrane receptor, appears to make binding to its ligand, NM23, possible [29]. CT and TD are highly conserved among species [25]. However, there are many transcriptional regulator elements in this region, which are not fully conserved between humans and mice [30]. Since the MUC-1-N subunit is imperfect with highly conserved variations [28], using a protein sequence homology analysis, it has been demonstrated that the CQCRRK sequence of MUC-1-C is fully conserved in dog and human proteins. Furthermore, dog MUC-1 CD shares a notable sequence homology of $83 \%$ with MUC-1 CD proteins of humans [31]. In HBC, known mutations of MUC-1 gene occurs as somatic changes within tumor genome [32]. On one hand, Carvajal-Agudelo et al. [33] found three single-nucleotide polymorphisms (SNPs) and two deletions (one in exon 7 and one in intron 6) in canine MUC-1. Furthermore, Carvajal-Agudelo et al. [33] found no significant correlation between MUC-1 expression and tumor grade or tumor type among CMTs; they found no correlation between MUC-1 and CMT incidence [33]. On the other hand, Manuali et al. showed that in CMT, MUC-1 expression is positively related to tumor grade; high MUC-1 in serum is found in grade II and III [24, 34]. MUC-1 overexpression is associated with poor prognosis in canine model [34].

\section{MUC-1 expression level and pattern of expression}

In normal breast epithelial cells, MUC-1 is expressed at low levels just on the apical surfaces [19]. There is a different expression pattern of MUC-1 in both human and canine breast tissues, as well as in normal breast epithelial and tumor cells $[14,35]$. Over the malignant transformation, the membrane expression of MUC-1 usually alters from apical to circumferential altogether with the loss of polarity of these epithelial cells, performing as anti-adhesive molecules, assisting the detachment of tumor cells, and thus increasing the metastatic and invasiveness of malignant cells [21]. The first study on MUC-1 expression in CMT was conducted in 2009 
[14]. In this study, apical cellular localization of MUC-1 was found in normal canine mammary gland tissue but not in adjacent mammary tumors, and no significant association between the tumor histological type and the level of MUC-1 expression was found; this could be due to small sample size [14]. Meanwhile, in human studies, there was an association between high histopathological grading and MUC-1 over-expression [18, 36]. In 2012, a significant correlation was found between the MUC-1 expression level and the histopathological grade of tumor malignancy in human studies [24]. In a more recent study on canine MUC-1 expression, different expression patterns were detected among different CMT types; 92.8\% were positive for cytoplasmic MUC-1 expression, $64.2 \%$ were positive for membrane MUC-1 expression, and 10.7\% were positive for nuclear MUC-1 expression [20]. Furthermore, it has been proved that MUC-1 expression in primary tumors of dogs with metastasis to a regional lymph node is significantly higher than in tumors of dogs without lymph node metastasis [20]. Study in estrogenreceptor positive $(E R+)$ human breast cancer have revealed that estrogen receptor $\alpha(E R \alpha)$ resides in estrogen-responsive elements of the MUC1 promoter and triggers MUC1 transcription [37]. In CMT, the presence of estrogen receptor was correlated with pathological characteristics of the cancer, and the presence of ER seems to correlate with the degree of differentiation [38]. In particular, a lower expression of ER $\alpha$ was related to a worse prognosis, a larger size and skin ulceration [39].

\section{MUC-1 signaling pathway}

MUC-1 involves in apoptotic response in cancer cells and $\mathrm{HBC}$, playing as a carcinogen in it [33]. The MUC-1-C transmembrane subunit interacts with receptor tyrosine kinases (RTKs), such as ErB2 and epidermal growth factor receptor (EGFR), at the cell surface and contributes to the activation of PI3K- Akt and mitogen-activated protein kinase (MEK)-extracellular signal-regulated kinase (ERK) downstream intracellular signaling pathways [31, 40]. MUC-1-C also contributes to activation of the Wnt/b-catenin cascade, signal transducer and activator of transcription (STAT), and nuclear factor- $\mathrm{kB}$ (NF- $\mathrm{kB}$ ) RelA pathways by localizing to the nucleus (Fig. 1) [40].

As far as MUC-1-C is highly conserved among species [25] and acts as signal transduction to downstream effectors [40], we expect the same behavior in both canine and human signaling activation of this antigen. As evidence, a recent study on the CMT cell line (CMT-7364) showed that MUC-1 overexpression promoted the activation of the PI3K/Akt signaling cascade [8]. Zhao et al. showed

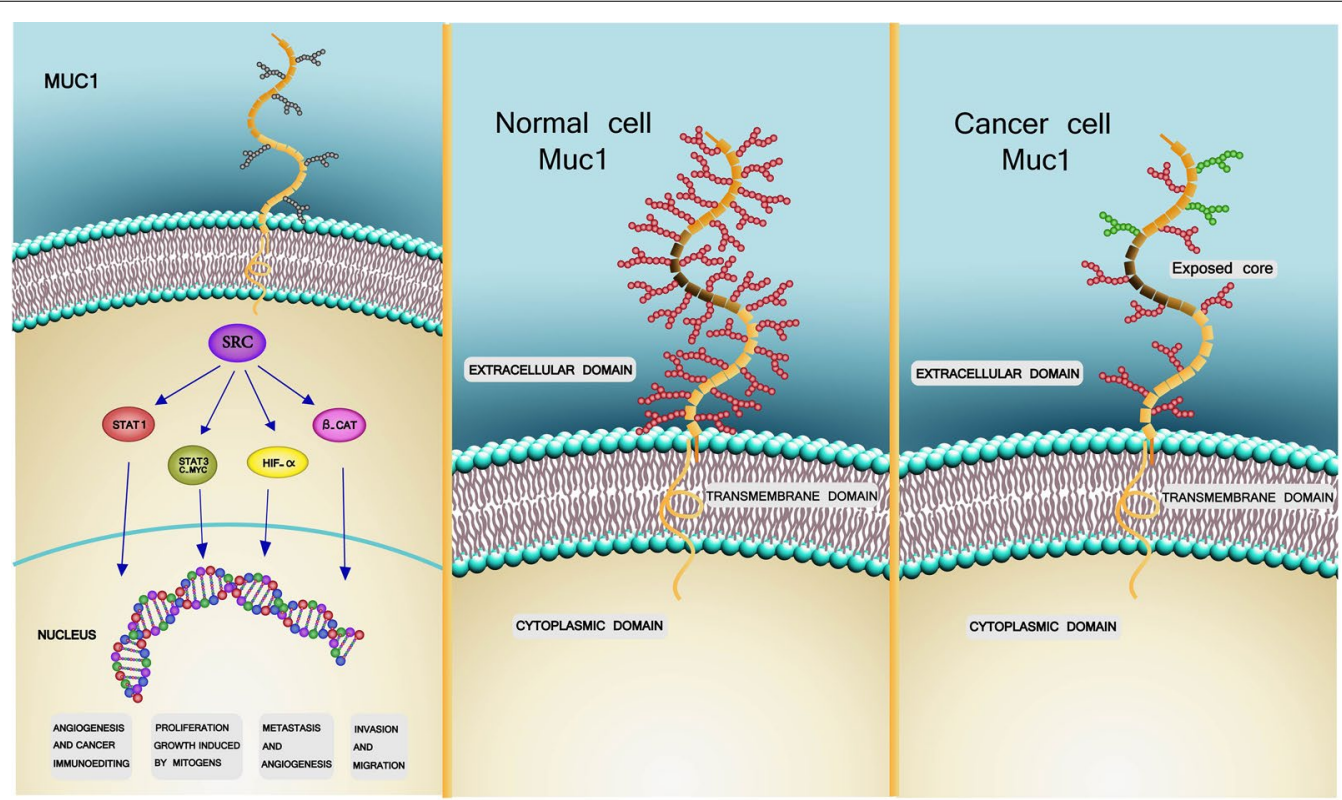

Fig. 1 A MUC1 belongs to mucin family and its role in normal cells is protection of beneath cells from physical damages. In contrast, MUC1 play a different and complicated role in cancer cells. MUC1 cytoplasmic domain has crosstalk with other molecules like STAT1, HIFa and so on. These molecules are involved in growth, proliferation, angiogenesis, migration, invasion and metastasis. Because MUC1 has interaction with aforementioned molecules, it is a potential target in cancer therapy. B As illustrated above, MUC1 is hyper-glycosylated in normal cells. The tandem repeat, the black part including the APDTRRPAD amino acid sequence, is under the carbohydrate and consequently hided. C In cancer cells not only there is less carbohydrate density, but also new carbohydrates appear. Meanwhile, tandem repeat which was under carbohydrates before, becomes exposed. Thus, it can be concluded that MUC1 has distinct structure in normal and cancer cells 
in their research that MUC-1 interacted with AKt and by subsiding disulfiram effects on cancer cells in CMT, made them resistant to disulfiram [8]. By downregulation of MUC-1, AKt signaling was suppressed and this result established the crosstalk between MUC-1 and Akt [8]. In addition, they showed that with the activation of this pathway, cell migration and proliferation were also substantially promoted following its overexpression [8].

\section{CA15-3 detections in peripheral blood of dog and human}

Circulating markers, such as glycoprotein substances, are measurable in blood and body fluids in both neoplastic and health conditions. They usually have higher levels of neoplasia and are known as tumor indicators and prognostic tools [4]. MUC-1-N truncates and sheds blood from breast cancer cell surfaces and is found in the serum of women with metastatic breast cancer at increased levels [40]. The soluble form of MUC-1 in peripheral blood is called CA15-3 [41], extremely expressing in human mammary malignancies, and is one of the best known and most broadly used serum tumor markers in women with breast cancer [24]. This marker also is used as a monitoring tool for response to treatment [42]. Furthermore, there is a correlation between the circulating CA15-3 level and histopathological grade of breast cancer [24]. Accumulating evidence suggests that this glycoprotein is a potential promising marker in canine medicine in addition to human medicine; therefore, here, we provided a summary of these pieces of evidence. For the first time, Clinton et al. [43] studied the diagnostic efficacy of dogs' serum CA15-3 and found 63\% diagnostic sensitivity and $80.64 \%$ specificity using the Centocor CA15-3 assay (Fujirebio Diagnostics, Centocor Inc), which is a solidphase radioimmunoassay (RIA). They used the 115D8 murine monoclonal antibody (mAb; as the capture antibody), besides the I125 labeled DF3 murine mAb; thus, it was concluded that CA15-3 could be considered the best tumor antigen regarding diagnostic aid and monitoring agent [43]. Five years later, using IMMULITE appliance and human kits (Medical System S.P.A, Genoa), Marchesi et al. [44] measured CA15-3 in serum and plasma of clinically healthy dogs/non-neoplastic diseases and dogs with clear tumor lesions other than mammary tumors. They concluded that the determination of CA15-3 could not differentiate among any of their study groups [44]. In another study, they performed the measurements using ADVIA Centaur by the direct chemiluminescence method and human kits (Bayer Immuno 1 CA15-3) and found it applicable for the determination of this marker in canine mammary oncology diagnostics [4]. They reported that subjects with serum CA15-3 levels higher than $1 \mathrm{U} / \mathrm{mL}$ were found to have mammary neoplasia based on pathology assessments [4]. In a study by Campos et al. [42], a significant positive correlation between mammary neoplasm staging and this serum marker was observed. Also, in agreement with the previous study, the serum concentration of CA15-3 was significantly less in dogs with no evidence of mammary tumors compared to dogs with benign and malignant mammary masses. Three years later, they also found a significant increase in serum CA15-3 in dogs with regional lymph node metastatic mammary neoplasms compared to those without metastasis and healthy dogs. In this study, authors used commercial solid-phase, non-competitive ELISA immunoassay kits [20]. In the most recent study on this field, this marker was analyzed using canine-specific kits of Bioassay Technology Laboratory, and it was found to be higher in dogs with mammary cancer compared to controls (tumor-free dogs) with high sensitivity, supporting the aforementioned studies [45]. These findings reflect the fact that this tumor marker could not only be used in dogs as a model for human studies but also is a promising diagnostic tool in canine oncology.

\section{Immunogenicity of MUC-1}

MUC-1 immunogenicity was examined in mice and human models [46]; the results showed that MUC-1 induced humoral and cellular immunity [47]. In the case of cellular immunity, cytotoxic lymphocyte (CTL) cells were isolated from BC patients, reacted to the MUC-1 tandem repeat $[46,48]$. Also, there is a humoral response to MUC-1; some patients with benign tumors had antiMUC-1 antibodies in their blood and showed a better prognosis [46]. Indeed, the results demonstrated that anti-MUC-1 immunoglobulin G (IgG) could bind to MUC-1 in some BC patients and mice models [46]. Numerous studies have been conducted to know which part of MUC-1 can induce immune response successfully. In this regard, the results have indicated that small epitopes of MUC-1 are highly immunogenic and targeted by mAbs [49]. Moreover, the CTL cells harvested from patients recognized the same epitope of MUC1, which was identified by mAbs $[49,50]$. Cancerous MUC-1 has short-chain O-glycosylation with low density; this structure allows the specific amino acid sequence to be exposed to the cell surface $[51,52]$. In this position, Tn and STn epitopes are accessible to the immune system [51]. This structure belongs to cancer cells uniquely and results in the discrimination of a mAb between normal and cancer cells [51].

On the other hand, autoantibodies are produced by humoral immunity against low levels of tumor-associated antigens as a very specific antibody response, supplying an early cancer detection tool [18, 22]. Anti-mucin immunoglobulins (i.e., MUC-1-IgG and MUC-1-IgM) 
are one of these circulating antibodies [18]. The complex of MUC-1 with these antibodies can also be found in the bloodstream [53]. When the MUC-1 level elevates in circulation, the anti-MUC-1 antibody titer decreases concurrently; this may be caused by their binding and formation of the antigen-antibody complex [53]. AntiMUC-1 autoantibodies have been observed in both HBC and CMT [22] which proves that MUC-1 induces immune response in human and dog. Although these autoantibodies have been known as breast cancer biomarkers, the problem is that autoantibodies against aberrantly glycosylated MUC-1 are also present in healthy people and benign diseases, which makes it hard to differentiate between cancer and healthy titers [18]. Although a higher titer of circulatory anti-MUC-1 antibodies was found in humans with breast cancer [22], to the best of our knowledge, the first and only study on autoantibodies against MUC-1 in canines was recently conducted in 2018 [22]. To demonstrate the diagnostic potential of several autoantibodies (such as MUC-1) in CMT, they took advantage of a high-throughput Luminex technique [22]. Their results showed that the anti-MUC-1 autoantibody assay had the highest sensitivity and specificity $(62.67 \%$ and $98 \%$, respectively) among other anti-TAA antibodies, including triosephosphate isomerase (TPI), phosphoglycerate mutase 1 (PGAM1), manganese superoxide dismutase (MnSOD), and avian myelocytomatosis viral oncogene homolog (c-Myc), to detect mammary tumors in dogs [22]. According to the therapeutic, immunogenicity, oncogenicity, epitope numbers, and expression level, the National Cancer Institute (NCI) ranked MUC-1 as the second target for clinical goals [54]. Mucins can block CTL, natural killer (NK) cells, and neutrophil activity and consequently inhibit anti-tumor immune response [49]. MUC-1 inhibits the binding of immune cells to their target and hinders the immunotherapy effectiveness in cancers with epithelial cells originally [49]. The reason is that MUC-1 is a self-antigen, even in its specific structure on cancer cells tolerance, which does not allow the immune system to respond properly [47]. Hence, some techniques have been used to improve immune response to MUC1 , especially increasing the efficacy of vaccines [47]. For instance, some researchers have used tumor-specific carbohydrates (inducing an immune response), which may lead to less tolerance to these tumor-specific carbohydrates in patients [47].

\section{Canine immune response to mammary tumors}

Studies show that immune subsets of human and canine are similar [10], furthermore, the immune response to mammary tumors shows many similarities in human and dogs. According to several research results, not only the composition of immune cells in tumor microenvironment in $\mathrm{HBC}$ and $\mathrm{CMT}$ is alike, but also results demonstrate a parallel expression pattern of immune molecules in both tumors [10]. In cellular level, CMT cells like HBC cells show high number of neutrophil cells [55], TAMs $[10,56]$, and CD $4+[12,55,56]$ cells which are associated with metastasis $[55,56]$. Furthermore, high number of plasma cells, macrophages, CD8+ are negatively related to metastasis [55]. Like HBC, in CMT M2 macrophages and $\mathrm{CD} 3+\mathrm{T}$ cells play a role in angiogenesis, in both HBC and CMT, M2 macrophages secrete VEGF [56]. In molecular level, the expression of programmed death-ligand 1 (PDL1) and PDL2 two co-inhibitory molecule- have been examine in CMT cells. PDL2 expression is reduced in CMT metastatic tumors, similar phenomenon happens for PDL1 expression in metastatic HBC [10]. Gal9 boosts anti-tumor immunity mediated Th1 cells in HBC and CMT [10]. Gal9 is a prognostic marker in both models and in human, gal9 expression is negatively related to distant metastasis, moreover, in CMT, gal9 expression in reduced in tumor cells [10]. IL-10 is a robust anti-inflammatory cytokine and is secreted by immune cells like T-reg cell and cancer cells, its expression is upregulated in HBC and CMT [10].

\section{The role of MUC-1 in cancer immunotherapy}

Immunotherapy is one of the newest strategies used in BC. Since there are some specific problems and complexity with other strategies of BC therapy (such as chemotherapy, radiotherapy, and surgery), the need for a new strategy made researchers propose immunotherapy [57, 58]. Different immunotherapy methods, including mAb, vaccine, chimeric antigen receptor (CAR) $T$ cells, have been investigated in BC. For example, trastuzumab is the most known agent that has been utilized in HER $2+B C$ patients and improved prognosis in these patients [59].

\section{Immunotherapy in canine tumors}

There are few examples of immunotherapy approaches which have been used in canine cancers like melanoma and lymphoma. Some of these approaches are used for treatment of canine cancers, while others have been under investigation in clinical phases. For example, anti$\mathrm{CD} 20 \mathrm{mAb}$ is used for treatment of lymphoma in dogs [12]. Moreover, dogs with metastatic melanoma have been treated by Indoleamine 2,3-dioxygenase (IDO) inhibitors in combination with radiotherapy [12]. On the other hand, cDNA vaccine- targeting tyrosinase- has been investigated in phase II and III clinical trial for oral melanoma [12]. Different settings of HBC immunotherapy have been under investigation, which target MUC-1. These settings include vaccine, mAb, CAR T cells, and combination immunotherapy. The following is a survey of these settings and their results. By reading the following 
survey, we should consider MUC-1 as a potent therapeutic target not only for HBC but also for CMT.

\section{Anti-MUC-1 vaccine}

Researchers have examined the effects of the MUC-1 subunit vaccine [keyhole limpet hemocyanin (KLH) and DETOX as an adjuvant] in BC patients [60]. The results of this study showed that this vaccine exploited CTLs restricted MHC-I response [60]. In different settings, the MUC-1 DNA vaccine and GM-CSF were evaluated in a murine model of $\mathrm{BC}$ [60]. The results demonstrated that this vaccine could reject tumors in mice [60]. The MUC-1 glycopeptide vaccine is another type of the MUC-1 vaccine that has been investigated. Tetanus toxin and sialylTn epitope of MUC-1 were injected into mice bearing BC and decreased tumor growth in them [60]. A MUC-1/ HER-2 chimer protein was examined in a BC mice model, and its results showed that humoral and cellular immune response was induced in these animals [54]. In this study, mice produced anti-MUC-1/HER-2 IgG and $T$ helper 1 response [54]. This vaccine increased cancer cells' necrosis and reduced lung metastasis [54]. The anti$\mathrm{STn}$ vaccine could induce IgG and IgM production in $\mathrm{BC}$ patients [49].

MUC-1 and toll-like receptor 7 (TLR-7) peptide in a murine model of $\mathrm{BC}$ were tested, and this vaccine induced CTL response and antibody-dependent cellular cytotoxicity (ADCC) and improved humoral immunity against MUC-1 + cancer cells [61]. This vaccine might inhibit tumor growth and recurrence and showed significant cytotoxicity in vivo [61]. A virus-like particle containing MUC-1 and survivin peptide was evaluated in a triple-negative breast cancer (TNBC) mice model, which specifically induced cytotoxicity and CTL response [62]. The conserved MUC-1 peptide and dendritic cells (DCs) induced immune response against tumor cells [51]. In a murine BC model, the MUC-1 liposome vaccine and interleukin 2 (IL-2) were investigated; it could induce interferon gamma (IFN- $\gamma$ ) secreting $\mathrm{T}$ cell, which target MUC-1 [46]. In a clinic, in order to overcome selftolerance to MUC-1, in a study, HLA-A0201-MUC-1 and allogenic $\mathrm{DC}$ were tested in $\mathrm{BC}$ patients, which could create CTL response in them [47]. In MUC-1 vaccines, different adjuvants have been investigated to boost immune response. For instance, MUC-1 and tetanus toxin elicit a stronger immune response compared to the synthetic MUC-1 peptide alone [63]. In TNBC patients, the MUC-1 peptide and poly polyinosinic:polycytidylic acid (ICLC; an immune stimulator) were under investigation in clinical phase [64]. Tan et al. [65], in phase I clinical trial, examined an adenoviral vector vaccine, which carried a MUC-1/CD-40 peptide. In clinical settings, researchers have investigated the efficacy of autologous DC, which carried MUC-1 complementary DNA (cDNA) in advanced BC patients [60]. The patients showed T CD8 + response, specifically bound to MUC-1 and secreted IFN- $\gamma$ [60]. In a clinical trial phase II study, sialyl-STn in conjugation with KLH was tested in BC patients, which showed no efficacy [60].

\section{Monoclonal antibody}

There is a long list of $\mathrm{mAb}$ and antibody fragments, which have been applied in BC [59]. These agents targeted different antigens (such as HER2, VEGF, and so on) and improved the overall survival and quality of life of $\mathrm{BC}$ patients [59]. Hence, it can be assumed that $\mathrm{mAb}$ and antibody fragments are the most successful immunotherapeutic agents in BC. Also, mAbs against MUC- 1 can be used as diagnostic and therapeutic agents in $\mathrm{BC}[49$, 51]. Most of the anti-MUC-1 mAbs react to cancerous tissue with high affinity and show low affinity to normal tissue [49]. Hence, it can be concluded that these mAbs target cancer cells specifically. There is a murine $\mathrm{mAb}$ that recognizes Tn and STn in MUC-1 [51]. Another $\mathrm{mAb}, \mathrm{SM}-3$, is an anti-MUC-1 mAb, which binds to MUC-1 specifically [66]. SM-3 and CTL cells recognize the same epitope of MUC-1 [66]. The clinical outcomes of anti-MUC-1 mAbs produced in mice have not been promising [51]. This failure was due to the source of these antibodies. On the other hand, it is possible that a humanized antibody could promote ADCC successfully [51]. Zhou et al. [52] produced a TAB004 anti-MUC-1 $\mathrm{mAb}$, which did not react to normal tissues and specifically bound to cancer cells. TAB004 targeted $95 \%$ of all MUC-1+ malignant tissue, including TNBC samples [52]. Panchamoorthy et al. [67] generated an anti-MUC1-C mAb and showed that it could bind to $\mathrm{BC}$ cell lines and induced cytotoxicity responses; it also showed the anti-tumor activity in mice bearing BC xenograft. They suggested that their $\mathrm{mAb}$ can be used as a drug conjugated $\mathrm{mAb}[67]$.

\section{Application of CAR T cells}

CAR T cells are T cells engineered TCR and are one of the immunotherapy strategies [58]. Because $T$ cells play a central role in the immune system, using them in cancer therapy is an interesting research area. Therefore, several groups worked on CAR T cells [51]; for example, in one study, anti-MUC-1 specific CAR T cells were produced and showed significant anti-tumor activity and cytotoxicity in TNBC in vivo and in vitro [52].

\section{Combination immunotherapy}

Using single-agent immunotherapy achieved success in BC; however, problems such as resistance appear [58]. To target cancer more effectively, combining different 
immunotherapy agents with distinct targets could be more useful [58]. In BC targeting, PD- 1 and CTLA-4 by mAbs were approved by the US Food and Drug Administration $[57,58]$. Considering the fact that MUC-1 has been examined in immunotherapy lately, MUC-1 has been introduced to the combination immunotherapy field in BC. There is a combination of the MUC-1 messenger RNA (mRNA) vaccine and anti-CTLA-4 targeting $\mathrm{mAb}$, which was tested in the TNBC animal model [68]. In this study, this combination strategy prevented tumor growth in tested mice.

\section{Conclusions and future perspectives}

Nowadays, using animals with spontaneously occurring tumors has received a great deal of attention in human studies as a proper cancer model and also to "translating clinical trials from human to veterinary oncology and back" [5]. The unique features of CMT, such as occurring spontaneously, size similarities, identical clinical stages, rapid growth, etc., have made this tumor an interesting choice for comparison studies. Another fascinating aspect of utilizing CMT in human studies is the potential to exhibit the same molecular characterizations in both species. In this aspect, MUC-1 is one the most thoroughly studied TAAs in human research, though narrowly assessed in canine and comparison investigations. Thus, in order to find a more promising potential of MUC-1 in diagnostic and therapeutic facets of comparison cancer research, here, we summarized the current knowledge about the different aspects of this biomarker in human and canine views.

Gene and protein structure similarities, as well as the level and pattern of expression, along with common signaling pathways, make canine MUC-1 a proper candidate for human studies. However, in canine oncology, the role of MUC-1 in tumors is less investigated and it is undeniable that there are also many challenges and risks that are preventing the practical using of this model. Lack of potent infrastructures which provide coordination among veterinary hospitals, researchers, oncologists and pharmaceutical companies are important points which have barricaded the potential application of such a model [7]. Identification of breed specific risk factors of CMT is also a necessity [7]. One recommendation is to conduct more studies on the subject of canine MUC-1 together with getting through the comparison research of MUC-1.

\footnotetext{
Abbreviations

MUC-1: Mucin-1; CMT: Canine mammary tumor; HBC: Human breast cancer; SNPs: Single-nucleotide polymorphisms; CD: Cluster of differentiation; CA: Cancer antigen; KL: Krebs von den Lungen; CD: Cytoplasmic domain; TD: Transmembrane domain; VNTR: Variable number of tandem repeats; RTKs: Receptor tyrosine kinases; EGFR: Epidermal growth factor receptor; MEK: Mitogen-activated protein kinase; ERK: Extracellular signal-regulated kinase;
}

STAT: Signal transducer and activator of transcription; ER+: Estrogen-receptor positive; MAB: Monoclonal antibody; CTL: Cytotoxic lymphocyte; TAA: Tumorassociated antigen; IgG: Immunoglobulin G; TPI: Triosephosphate isomerase; PGAM: Phosphoglycerate mutase; MnSOD: Manganese superoxide dismutase; Myc: Myelocytomatosis; NCl: National Cancer Institute; NK: Natural killer; PDL: Programmed death-ligand; CAR: Chimeric antigen receptor; IDO: Indoleamine 2,3-dioxygenase; TLR: Toll-like receptor; ADCC: Antibody-dependent cellular cytotoxicity; TNBC: Triple-negative breast cancer; DC: Dendritic cells; IFN: Interferon gamma; cDNA: Complementary DNA; FDA: Food and Drug Administration; mRNA: Messenger RNA.

\section{Acknowledgements}

Not applicable.

\section{Authors' contributions}

RV performed the literature search, collected the data and wrote the manuscript. MS, AH and khB collected the data and wrote the manuscript and designed figures. MRE collected the data, and reviewed manuscript. LF and ZKh reviewed manuscript. All authors contributed to the preparation of the manuscript. All authors read and approved the final manuscript.

\section{Funding}

This article has not received any funding.

Availability of data and materials

Not applicable.

\section{Declarations}

Ethics approval and consent to participate

Not applicable.

\section{Consent for publication}

All authors are in agreement with the publication of the manuscript.

\section{Competing interests}

The authors declare that they have no competing interest.

\section{Author details}

${ }^{1}$ Recombinant Proteins Department, Breast Cancer Research Center, Motamed Cancer Institute, ACECR, No.146, South Gandi Ave, Vanak Sq, Tehran, Iran.

${ }^{2}$ Department of Clinical Pathology, Faculty of Veterinary Medicine, University of Tehran, Tehran, Iran. ${ }^{3}$ Department of Immunology, School of Medicine, Iran University of Medical Sciences, Tehran, Iran. ${ }^{4}$ Department of Surgery and Radiology, Faculty of Veterinary Medicine, University of Tehran, Tehran, Iran.

Received: 22 August 2021 Accepted: 8 December 2021

Published online: 09 January 2022

\section{References}

1. Porrello A, Cardelli P, Spugnini E. Oncology of companion animals as a model for humans. An overview of tumor histotypes. J Exp Clin Cancer Res. 2006;25(1):97-105

2. Vail DM, Macewen EG. Spontaneously occurring tumors of companion animals as models for human cancer. Cancer Invest. 2000;18(8):781-92.

3. Owen L. A comparative study of canine and human breast cancer. Invest Cell Pathol. 1979;2(4):257.

4. Marchesi M, Manuali E, Pacifico E, Ferri C, Romagnoli M, Mangili V, et al. Cancer antigen 15/3: possible diagnostic use in veterinary clinical oncology. Preliminary study. Vet Res Commun. 2010;34(1):103-6.

5. Fürdös I, Fazekas J, Singer J, Jensen-Jarolim E. Translating clinical trials from human to veterinary oncology and back. J Transl Med. 2015;13(1):1-7.

6. Bird RC, Deinnocentes P, Church Bird AE, van Ginkel FW, Lindquist J, Smith BF. An autologous dendritic cell canine mammary tumor hybrid-cell fusion vaccine. Cancer Immunol Immunother. 2011;60(1):87-97. 
7. Gray M, Meehan J, Martínez-Pérez C, Kay C, Turnbull AK, Morrison LR, et al. Naturally-occurring canine mammary tumors as a translational model for human breast cancer. Front Oncol. 2020;10:617.

8. Zhao Y, Lin Z, Lin Z, Zhou C, Liu G, Lin J, et al. Overexpression of mucin 1 suppresses the therapeutical efficacy of disulfiram against canine mammary tumor. Animals. 2021;11(1):37.

9. Valdivia G, Alonso-Diez Á, Pérez-Alenza D, Peña L. From conventional to precision therapy in canine mammary cancer: a comprehensive review. Front Vet Sci. 2021. https://doi.org/10.3389/fvets.2021.623800.

10. Bujak JK, Szopa IM, Pingwara R, Kruczyk O, Krzemińska N, Mucha J, et al. The expression of selected factors related to T lymphocyte activity in canine mammary tumors. Int J Mol Sci. 2020;21(7):2292.

11. Raposo TP, Arias-Pulido H, Chaher N, Fiering SN, Argyle DJ, Prada J, et al. Comparative aspects of canine and human inflammatory breast cancer. Semin Oncol. 2017. https://doi.org/10.1053/j.seminoncol.2017. 10.012

12. Park JS, Withers SS, Modiano JF, Kent MS, Chen M, Luna JI, et al. Canine cancer immunotherapy studies: linking mouse and human. J Immunother Cancer. 2016:4(1):1-11.

13. Abdelmegeed SM, Mohammed S. Canine mammary tumors as a model for human disease. Oncol Lett. 2018;15(6):8195-205.

14. de Oliveira JT, Pinho SS, de Matos AJ, Hespanhol V, Reis CA, Gärtner F. MUC1 expression in canine malignant mammary tumours and relationship to clinicopathological features. Vet J. 2009;182(3):491-3.

15. Albrecht H, Carraway KL III. MUC1 and MUC4: switching the emphasis from large to small. Cancer Biother Radiopharm. 2011;26(3):261-71.

16. Nemunaitis J, Bedell C, Klucher K, Vo A, Whiting S. Phase 1 dose escalation of ONT-10, a therapeutic MUC1 vaccine, in patients with advanced cancer. J Immunother Cancer. 2013. https://doi.org/10.1186/ 2051-1426-1-S1-P240.

17. Hamanaka Y, Suehiro Y, Fukui M, Shikichi K, Imai K, Hinoda Y. Circulating anti-MUC1 IgG antibodies as a favorable prognostic factor for pancreatic cancer. Int J Cancer. 2003;103(1):97-100.

18. Guillen-Poza PA, Sánchez-Fernández EM, Artigas G, García Fernández JM, Hinou H, Ortiz Mellet C, et al. Amplified detection of breast cance autoantibodies using MUC1-based Tn antigen mimics. J Med Chem. 2020;63(15):8524-33.

19. Jing $X$, Liang H, Hao C, Yang X, Cui X. Overexpression of MUC1 predicts poor prognosis in patients with breast cancer. Oncol Rep. 2019:41(2):801-10.

20. Campos LC, Silva JO, Santos FS, Araújo MR, Lavalle GE, Ferreira E, et al. Prognostic significance of tissue and serum HER2 and MUC1 in canine mammary cancer. J Vet Diagn Invest. 2015;27(4):531-5.

21. Hollingsworth MA, Swanson BJ. Mucins in cancer: protection and control of the cell surface. Nat Rev Cancer. 2004;4(1):45-60.

22. Hussain S, Saxena S, Shrivastava S, Arora R, Singh RJ, Jena SC, et al. Multiplexed autoantibody signature for serological detection of canine mammary tumours. Sci Rep. 2018;8(1):1-14.

23. Klopfleisch R, Lenze $D$, Hummel M, Gruber A. The metastatic cascade is reflected in the transcriptome of metastatic canine mammary carcinomas. Vet J. 2011;190(2):236-43.

24. Manuali E, De Giuseppe A, Feliziani F, Forti K, Casciari C, Marchesi MC, et al. CA 15-3 cell lines and tissue expression in canine mammary cancer and the correlation between serum levels and tumour histological grade. BMC Vet Res. 2012;8(1):1-10

25. Brayman M, Thathiah A, Carson DD. MUC1: a multifunctional cell surface component of reproductive tissue epithelia. Reprod Biol Endocrinol. 2004;2:4

26. Lacunza E, Bara J, Segal-Eiras A, Croce MV. Expression of conserved mucin domains by epithelial tissues in various mammalian species. Res Vet Sci. 2009;86(1):68-77.

27. Nath S, Mukherjee P. MUC1: a multifaceted oncoprotein with a key role in cancer progression. Trends Mol Med. 2014;20(6):332-42.

28. Ren J, Bharti A, Raina D, Chen W, Ahmad R, Kufe D. MUC1 oncoprotein is targeted to mitochondria by heregulin-induced activation of c-Src and the molecular chaperone HSP90. Oncogene. 2006;25(1):20-31.

29. Hikita ST, Kosik KS, Clegg DO, Bamdad C. MUC1* mediates the growth of human pluripotent stem cells. PloS ONE. 2008;3(10): e3312.

30. Carson DD. The cytoplasmic tail of MUC1: a very busy place. Sci Signal. 2008; 1(27):pe35.
31. Raina D, Agarwal P, Lee J, Bharti A, McKnight CJ, Sharma P, et al. Characterization of the MUC1-C cytoplasmic domain as a cancer target. PLoS ONE. 2015;10(8): e0135156.

32. Van der Merwe N, Peeters AV, Pienaar FM, Bezuidenhout J, Van Rensburg SJ, Kotze MJ. Exome sequencing in a family with luminal-type breast cancer underpinned by variation in the methylation pathway. Int J Mol Sci. 2017:18(2):467.

33. Carvajal-Agudelo JD, Giraldo-Chalarca L, Cortes-Mera DM, Ossa-López PA, Morales-Álvarez ED, Rivera-Páez FA. Detection of single nucleotide polymorphisms (SNPs) in HER2, MUC1, ESR1, and BRCA1 genes associated with canine mammary cancer. Vet stn. 2021. https://doi.org/10.46419/vs. 52.5.1.

34. Kaszak I, Ruszczak A, Kanafa S, Kacprzak K, Król M, Jurka P. Current biomarkers of canine mammary tumors. Acta Vet Scand. 2018;60(1):1-13.

35. Rahn JJ, Dabbagh L, Pasdar M, Hugh JC. The importance of MUC1 cellular localization in patients with breast carcinoma: an immunohistologic study of 71 patients and review of the literature. Cancer. 2001;91(11):1973-82.

36. Li Y-s, Kaneko M, Sakamoto DG, Takeshima Y, Inai K. The reversed apical pattern of MUC1 expression is characteristics of invasive micropapillary carcinoma of the breast. Breast Cancer. 2006;13(1):58-63.

37. Zaretsky JZ, Barnea I, Aylon Y, Gorivodsky M, Wreschner DH, Keydar I. MUC1 gene overexpressed in breast cancer: structure and transcriptional activity of the MUC1 promoter and role of estrogen receptor alpha (ERa) in regulation of the MUC1 gene expression. Mol Cancer. 2006;5(1):1-14.

38. MacEwen E, Patnaik A, Harvey H, Panko W. Estrogen receptors in canine mammary tumors. Can Res. 1982;42(6):2255-9.

39. De Las Mulas JM, Ordás J, Millán M, Chacón F, De Lara M, De Los Monteros $A E$, et al. Immunohistochemical expression of estrogen receptor $\beta$ in normal and tumoral canine mammary glands. Vet Pathol. 2004;41(3):269-72.

40. Kufe DW. MUC1-C oncoprotein as a target in breast cancer: activation of signaling pathways and therapeutic approaches. Oncogene. 2013;32(9):1073-81.

41. Choi JW, Moon BI, Lee JW, Kim HJ, Jin Y, Kim HJ. Use of CA15-3 for screening breast cancer: An antibody-lectin sandwich assay for detecting glycosylation of CA15-3 in sera. Oncol Rep. 2018;40(1):145-54.

42. Campos LC, Lavalle GE, Estrela-Lima A, de Faria JCM, Guimaraes JE, Dutra AP, et al. CA15.3, CEA and LDH in dogs with malignant mammary tumors. J Vet Intern Med. 2012;26(6):1383-8.

43. Clinton SR, Beason KL, Bryant S, Johnson JT, Jackson M, Wilson C, et al. A comparative study of four serological tumor markers for the detection of breast cancer. J Miss Acad Sci. 2002;47(2):126.

44. Marchesi M, Conti M, Pieramati C, Mangili V, Fruganti G. Assessment and behavior of alphafetoprotein (AFP), antigen cancer 15/3 (CA 15/3), carcinembryonal antigen (CEA) in clinical oncology of the dog: preliminary study. Vet Res Commun. 2007;31(1):301-4.

45. Baba OK, Sood NK, Gupta K. Clinical evaluation of glycoproteins and inflammatory cytokines in the serum of dogs affected with canine mammary cancer. Proc Natl Acad Sci India Sec B Biol Sci. 2019;89(4):1465-9.

46. Lakshminarayanan V, Thompson P, Wolfert MA, Buskas T, Bradley JM, Pathangey $L B$, et al. Immune recognition of tumor-associated mucin MUC1 is achieved by a fully synthetic aberrantly glycosylated MUC1 tripartite vaccine. Proc Natl Acad Sci. 2012;109(1):261-6.

47. Pathangey LB, Lakshminarayanan V, Suman VJ, Pockaj BA, Mukherjee P, Gendler SJ. Aberrant glycosylation of anchor-optimized MUC1 peptides can enhance antigen binding affinity and reverse tolerance to cytotoxic $T$ lymphocytes. Biomolecules. 2016;6(3):31.

48. Apostolopoulos V, Pietersz GA, Xing P-X, Lees C, Michael M, Bishop J, et al. The immunogenicity of MUC1 peptides and fusion protein. Cancer Lett. 1995;90(1):21-6.

49. Apostolopoulos V, McKenzie IF. Cellular mucins: targets for immunotherapy. Crit Rev Immunol. 2017. https://doi.org/10.1615/CritRevImmunol. v37.i2-6.110.

50. Jerome KR, Barnd DL, Boyer CM, Taylor-Papadimitriou J, McKenzie IF, Bast $\mathrm{RC} \mathrm{Jr}$, et al. Adenocarcinoma reactive cytotoxic T lymphocytes recognize an epitope present on the protein core of epithelial mucin molecules. Cell Immun Immunother Cancer. 1990;321:328.

51. Gong Y, Klein Wolterink RG, Gulaia V, Cloosen S, Ehlers FA, Wieten L, et al. Defucosylation of tumor-specific humanized anti-MUC1 monoclonal antibody enhances NK cell-mediated anti-tumor cell cytotoxicity. Cancers. 2021:13(11):2579. 
52. Zhou R, Yazdanifar M, Roy LD, Whilding LM, Gavrill A, Maher J, et al. CAR $T$ cells targeting the tumor MUC1 glycoprotein reduce triple-negative breast cancer growth. Front Immunol. 2019;10:1149.

53. Croce MV, Isla-Larrain MT, Demichelis SO, Segal-Eiras A, Gori JR, Price MR. Tissue and serum MUC1 mucin detection in breast cancer patients. Breast Cancer Res Treat. 2003;81(3):195-207.

54. Gheybi E, Salmanian AH, Fooladi AAI, Salimian J, Hosseini HM, Halabian $R$, et al. Immunogenicity of chimeric MUC1-HER2 vaccine against breast cancer in mice. Iran J Basic Med Sci. 2018;21(1):26.

55. Markkanen E. Know thy model: charting molecular homology in stroma reprogramming between canine and human mammary tumors. Front Cell Dev Biol. 2019;7:348.

56. Carvalho MI, Silva-Carvalho R, Pires I, Prada J, Bianchini R, Jensen-Jarolim E, et al. A comparative approach of tumor-associated inflammation in mammary cancer between humans and dogs. BioMed Res Int. 2016. https://doi.org/10.1155/2016/4917387.

57. Barzaman K, Karami J, Zarei Z, Hosseinzadeh A, Kazemi MH, MoradiKalbolandi S, et al. Breast cancer: biology, biomarkers, and treatments. Int Immunopharmacol. 2020;84: 106535.

58. Barzaman K, Samadi M, Moradi-Kalbolandi S, Majidzadeh-A K, Salehi M, Jalili N, et al. Development of a recombinant anti-VEGFR2-EPCAM bispecific antibody to improve antiangiogenic efficiency. Exp Cell Res. 2021. https://doi.org/10.1016/j.yexcr.2021.112685.

59. Moradi-Kalbolandi S, Hosseinzade A, Salehi M, Merikhian P, Farahmand L. Monoclonal antibody-based therapeutics, targeting the epidermal growth factor receptor family: from herceptin to Pan HER. J Pharm Pharmacol. 2018;70(7):841-54.

60. Gao T, Cen Q, Lei H. A review on development of MUC1-based cancer vaccine. Biomed Pharmacother. 2020;132: 110888.

61. Liu Y, Tang L, Gao N, Diao Y, Zhong J, Deng Y, et al. Synthetic MUC1 breast cancer vaccine containing a Toll-like receptor 7 agonist exerts antitumor effects. Oncol Lett. 2020;20(3):2369-77.

62. Campbell K, Young VL, Donaldson BC, Woodall MJ, Shields NJ, Walker GF, et al. Delivering two tumour antigens survivin and mucin-1 on virus-like particles enhances anti-tumour immune responses. Vaccines. 2021;9(5):463.

63. Glaffig M, Stergiou N, Schmitt E, Kunz H. Immunogenicity of a fully synthetic MUC1 glycopeptide antitumor vaccine enhanced by poly $(1: C)$ as a TLR3-activating adjuvant. ChemMedChem. 2017;12(10):722-7.

64. Zhang D, Liu X, Gao J, Sun Y, Liu T, Yan Q, et al. The role of epithelial cell adhesion molecule $\mathrm{N}$-glycosylation on apoptosis in breast cancer cells. Tumor Biol. 2017;39(3):1010428317695973.

65. Tan TJY, Chia JWK, Chong H-S, Li X, Tan SH, Hopkins R, et al. First-in-man study of Ad-sig-hMUC1/ecdCD40L vaccine for immunotherapy of MUC1 overexpressing epithelial cancers. Journal of Clinical Oncology. 2018:36:3098

66. Quinlin I, Burnside J, Dombrowski K, Phillips C, Dolby N, Wright S. Context of MUC1 epitope: immunogenicity. Oncol Rep. 2007;17(2):453-6.

67. Panchamoorthy G, Jin C, Raina D, Bharti A, Yamamoto M, Adeebge D, et al. Targeting the human MUC1-C oncoprotein with an antibody-drug conjugate. JCl Insight. 2018. https://doi.org/10.1172/jci.insight.99880.

68. Liu L, Wang Y, Miao L, Liu Q, Musetti S, Li J, et al. Combination immunotherapy of MUC1 mRNA nano-vaccine and CTLA-4 blockade effectively inhibits growth of triple negative breast cancer. Mol Ther. 2018;26(1):45-55

\section{Publisher's Note}

Springer Nature remains neutral with regard to jurisdictional claims in published maps and institutional affiliations. 